\title{
Viewpoint
}

\section{Fire in a Quantum Mechanical Forest}

\author{
Je-Geun Park \\ Center for Functional Interfaces of Correlated Electron Systems, Institute for Basic Science, Seoul National University, \\ Seoul 151-747, Korea and \\ FPRD, Department of Physics \& Astronomy, Seoul National University, Seoul 151-747, \\ Korea
}

\section{Carley Paulsen}

Institut Néel CNRS/UJF UPR2940, 38042 Grenoble cedex 9, France

Published May 13, 2013

\begin{abstract}
A new experimental scheme allows the controlled generation and observation of magnetic avalanches, a cascade of spin flips that propagates through a magnetic sample like a three-dimensional fire.
\end{abstract}

Subject Areas: Magnetism, Materials Science

\author{
A Viewpoint on: \\ Onset of a Propagating Self-Sustained Spin Reversal Front in a Magnetic System \\ P. Subedi, S. Vélez, F. Macià, S. Li, M. P. Sarachik, J. Tejada, S. Mukherjee, G. Christou, and A. D. Kent \\ Phys. Rev. Lett. 110, 207203 (2013) - Published May 13, 2013
}

In a magnetized material, a phenomenon akin to an avalanche can sometimes occur: one spin, or perhaps just a few, can suddenly flip, and thereby spark a cascade of spin flips that fully reverses the sample magnetization. Such "magnetic avalanches" have been observed in a wide variety of systems at low temperature (such as ferromagnets, superconductors, as well as disordered systems like spin glasses and spin ices). These phenomena are sometimes considered a nuisance, since they may lead to instabilities that are detrimental to experiments and applications. Yet they can be the manifestation of complex quantum-mechanical effects and may offer a controllable platform to study other avalanche processes, such as the propagation of combustion in a material on fire. In Physical Review Letters, Pradeep Subedi, at New York University, and colleagues report on a new way to control and measure such avalanches in $\mathrm{Mn}_{12}$-acetate [1, a prototypical molecular nanomagnet. Thanks to a novel experimental setup, the authors were able to clearly pinpoint the conditions under which the magnetic energy stored in a sample is released via an avalanche of spin flips that can be viewed as a "magnetic deflagration."

The physics of magnetic avalanches can be different in each magnetic system, but certain common features can be identified. Generally, avalanches occur in magnetically bistable systems, in which two spin states (spin up and spin down) are separated by a large energy barrier that cannot be easily overcome by thermal activation. In a magnetized material, one of the states has lower energy and is preferentially occupied. As an applied magnetic field is varied, the other state may become the lowest energy state. While the barrier can keep the system

DOI: $10.1103 /$ Physics.6.55

URL: http://link.aps.org/doi/10.1103/Physics.6.55 in the initial spin state for some time (i.e., the state is metastable), this works only up to a certain point: once a spin overcomes the barrier, the stored magnetic energy is released, and can be transferred to neighboring spins, raising their temperatures. This can, in turn, make it easier to overcome further barriers, triggering a runaway chain of spin flips.

Molecular nanomagnets, chemically synthesized magnetic materials whose constituent units are molecules with a large net spin, are particularly suitable for the study of magnetic avalanches. The molecular crystal studied by the authors is based on $\mathrm{Mn}_{12}$-acetate, a diskshaped, organic compound with 12 embedded Mn ions that give the molecule a giant net spin of $S=10$. At low temperature, the system can be thought of as an ensemble of identical and weakly interacting nanomagnets. Its large uniaxial anisotropy, due to the molecule's tetragonal symmetry, gives rise to the large energy barrier for the reversal of the spin direction along this axis. The most remarkable discovery in this compound was the observation by Novak and Sessoli that, at certain values of the applied field, spin reversal could take place by quantum tunneling through the energy barrier, in contrast to the much slower relaxation by thermal activation over the barrier [2].

In 1994, while investigating quantum tunneling in $\mathrm{Mn}_{12}$-acetate, we observed avalanches when sweeping the amplitude of the magnetic field [3]. Such avalanches completely reversed the sample magnetization within a few milliseconds and were accompanied by simultaneous temperature spikes. These suggested that the avalanches were driven by the release of the magnetic-field-induced 
Zeeman energy stored in the spins. Our calculations indicated that when one spin flips, the local sample temperature could rise from $\sim 100$ millikelvin to as much as 10 kelvin, which would explain how a very fast relaxation could take place and trigger the avalanches.

Inspired by Norman Maclean's book Young Men and Fire, we likened this process to the propagation of a fire in a three dimensional forest. The book reports Maclean's forensic analysis of the 1949 Mann Gulch fire in Montana that led to the death of 13 "smoke-jumpers," parachuted in to fight the fire. Under the conditions encountered on that extremely dry and hot August day, a fire can propagate at incredible speeds and in unpredictable ways. One of the theories to explain the tragedy was that, in its early stages, the fire spread from tree crown to tree crown: the top of the trees burned so intensely that the heat could cause the ignition in a nearby tree crown. Similarly, magnetic avalanches in $\mathrm{Mn}_{12}$-acetate could be modeled as a quantum mechanical analog of a three-dimensional forest fire, in which trees could be replaced by single $\mathrm{Mn}_{12^{-}}$ acetate molecules: spin "sticks" with $S=10$, which can transfer their Zeeman energy to neighboring spins when flipping.

A number of research groups developed strategies to experimentally study magnetic avalanches, delivering a number of key results. By gluing an array of magnetic sensors to a sample, Suzuki et al. observed that the avalanche propagated as a thin interface between regions of opposite magnetization, with front speeds as large as 15 meters per second 4 . The authors described the phenomenon as a magnetic "deflagration," in analogy with the propagation of a flame front through a combustible material. Hernandez et al. highlighted the role of quantum tunneling [5] by demonstrating that the avalanche speeds exhibited maxima at values of the magnetic field for which the tunneling across the spin-up/spin-down energy barrier became resonant. Further work showed that the application of perpendicular and parallel magnetic fields (with respect to the easy axis) amounts to controlling the tunneling probability and the barrier height. These results led to the development of the first theories of magnetic deflagration [6].

Now, Subedi et al. 1 have developed an innovative setup in which a heater is glued to one end of the sample. They bias the sample with various combinations of parallel and perpendicular fields and light a "match" at one end of the sample (by applying a heat pulse). They then follow the propagation of the spin-reversal front throughout the sample (see Fig. 11). The results reveal two distinct regimes that depend on the combination of the experimental parameters: a slow, thermally driven magnetic relaxation and a rapid magnetic deflagration. Building on previous theories, the authors propose a simple model that captures the observed experimental features. The model involves a competition between two processes: the release of stored Zeeman energy (occurring when spins flip, increasing the temperature) and thermal diffusion (which lets this energy leak out to
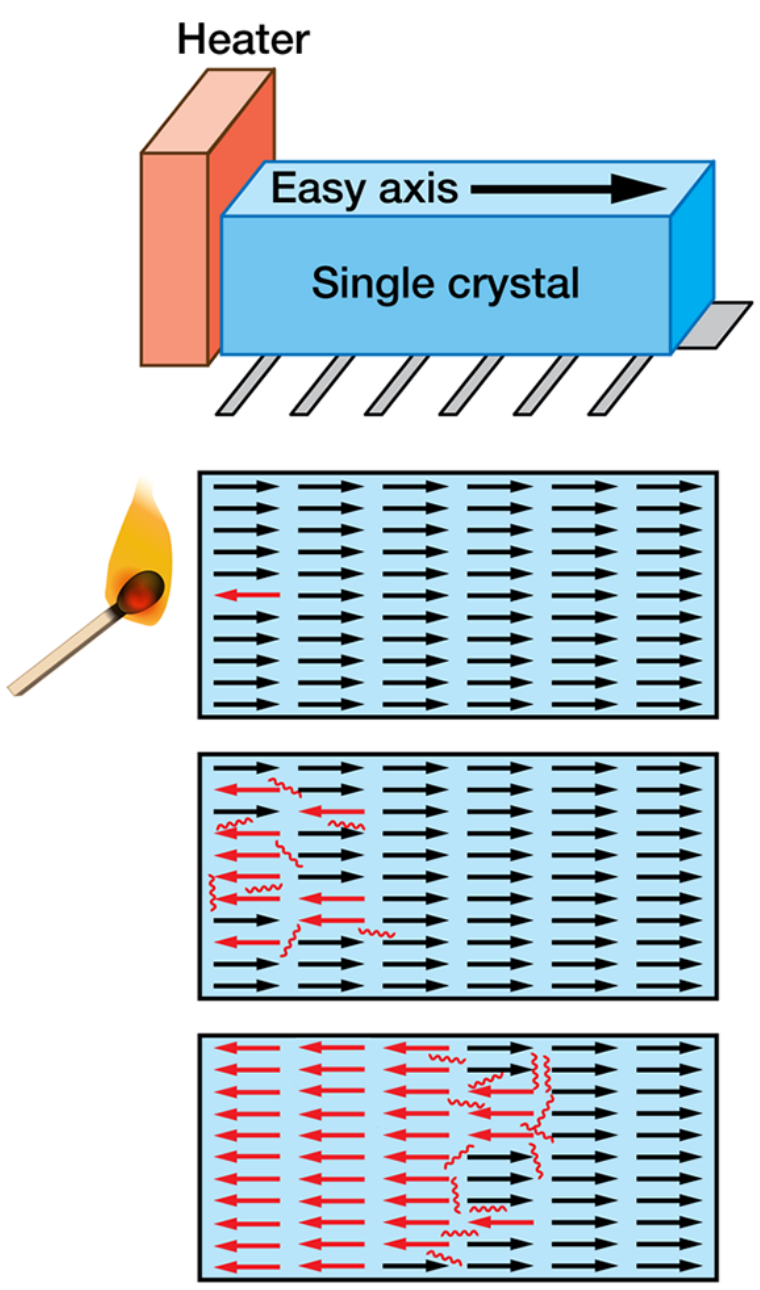

FIG. 1: (a) Schematic of the setup developed by Subedi et al. 1]: A heater triggers a magnetic avalanche in a single $\mathrm{Mn}_{12}$-acetate crystal, and magnetic sensors monitor the propagation of the spin reversal front across the sample. (b)-(d) Schematic of an avalanche in $\mathrm{Mn}_{12}$-acetate: (b) impulsive heating causes one spin to flip, releasing its Zeeman energy to a few neighboring spins; (c) these spins flip, heating their neighbors; (d) the process repeats itself in cascade and a magnetic "deflagration" results. (APS/Alan Stonebraker)

the surrounding cold bath, reducing the temperature). Experimentally, the first contribution can be regulated by choosing appropriate values of the magnetic fields (perpendicular and parallel). If it becomes sufficiently large, it can overwhelm the diffusion term. This makes the system unstable and raises the temperature rapidly, leading to deflagration. As an analogy, one can think of a leaky bucket in a status of precarious balance: If water is poured in slowly, it will leak out gradually. But if the water is dumped in too fast, the water will rise and the bucket will tip over.

The method of Subedi et al. provides detailed information on the exact conditions that ignite a fire in a $\mathrm{Mn}_{12}$ 
spin forest, allowing them to ignite the deflagration locally and to follow the macroscopic dynamics of its propagation. Future experiments may expand our capability of probing, in a time-resolved fashion, the microscopic dynamics of this nonequilibrium process: techniques like $\mathrm{x}$-ray diffraction may be able to capture a glimpse of the propagating front with atomic resolution. It is also time to look beyond $\mathrm{Mn}_{12}$-acetate, in order to develop a more general understanding of deflagration in magnetic systems. Approaches similar to that used by Subedi et al. may be applied to investigate other molecular magnets or entirely different systems such as the pyrochlore spin-ice $\mathrm{Dy}_{2} \mathrm{Ti}_{2} \mathrm{O}_{7}[7]$, in which different physics might be at work due to the strong spin correlation that gives rise to a frustrated ground state and to the emergence of monopoles. Plenty more magnetic fires may need to be lit before sufficient light is shed on the complex physics of magnetic deflagration.

Correction (16 May 2013): Paragraph 6, sentences 2 and 3 , the original article omitted the description of the key results of Ref. [4]. A brief explanation has been added.

Correction (22 May 2013): The figure has been corrected to indicate that the spins point parallel to the easy axis.

\section{References}

[1] P. Subedi, S. Vélez, F. Macià, S. Li, M. P. Sarachik, J. Tejada, S. Mukherjee, G. Christou, and A. D. Kent, "Onset of a Propagating Self-Sustained Spin Reversal Front in a Magnetic System," Phys. Rev. Lett. 110, 207203 (2013).

[2] M. A. Novak and R. Sessoli, "AC Susceptibility Relaxation Studies on a Manganese Organic Cluster Compound: $\mathrm{Mn}_{12^{-}}$ Ac," in Quantum Tunneling of Magnetization: QTM '94, edited by L. Gunther and B. Barbara (Kluwer Academic, Boston, 1995), pp. 171-188.

[3] C. Paulsen and J. G. Park, "Evidence for Quantum Tunneling of the Magnetization in $\mathrm{Mn}_{12}$-Ac," in Quantum Tunneling of Magnetization: QTM' 94, edited by L. Gunther and B. Barbara (Kluwer Academic, Boston 1995), pp. 189-207.

[4] Y. Suzuki et al., "Propagation of Avalanches in $\mathrm{Mn}_{12}$-Acetate: Magnetic Deflagration," Phys. Rev. Lett. 95, 147201 (2005).

[5] A. Hernandez-Minguez, J. M. Hernandez, F. Macia, A. GarciaSantiago, J. Tejada, and P. V. Santos, "Quantum Magnetic Deflagration in $\mathrm{Mn}_{12}$ Acetate," Phys. Rev. Lett. 95, 217205 (2005).

[6] D. A. Garanin and E. M. Chudnovsky, "Theory of Magnetic Deflagration in Crystals of Molecular Magnets," Phys. Rev. B 76, 054410 (2007).

[7] D. Slobinsky, C. Castelnovo, R. A. Borzi, A. S. Gibbs, A. P. Mackenzie, R. Moessner, and S. A. Grigera, "Unconventional Magnetization Processes and Thermal Runaway in Spin-Ice Dy2Ti2O7," Phys. Rev. Lett. 105, 267205 (2010).

\section{About the Authors}

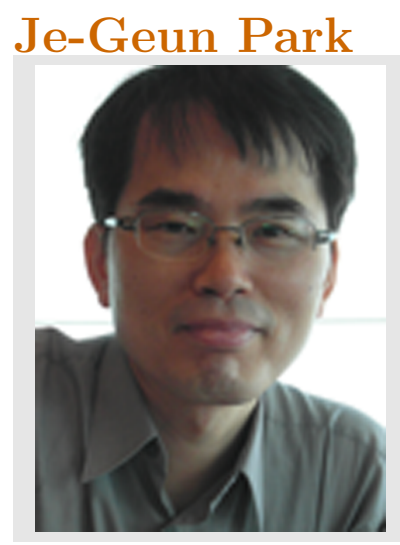

Je-Geun Park is professor of Department of Physics and Astronomy, Seoul National University, and group leader of Center for Functional Interfaces of Correlated Electron Systems, Institute for Basic Science, Korea. He received his Ph.D. from Imperial College London, and his research covers wide areas of strongly correlated electron systems. He has worked at CNRS in Grenoble, Birkbeck College-University of London, Inha University, and Sungkyunkwan University.

\section{Carley Paulsen}

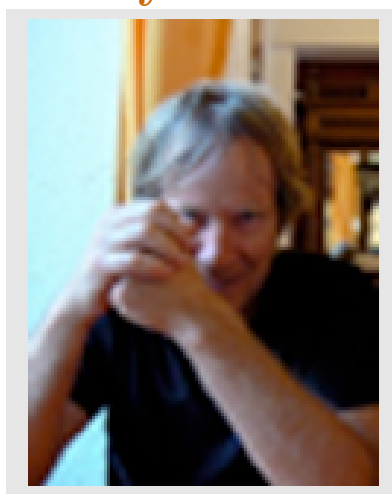

Carley Paulsen is Directeur de Recherche at the Institut Néel, CNRS, Grenoble. He received his Ph.D. from New York University and had postdoc positions at the Pontifícia Universidade Católica in Rio de Janeiro, Brazil, the Centre de Recherche sur les Très Basses Températures in Grenoble, Commissariat à l'Energie Atomique, in Saclay, France, and at Hokkaido University in Japan. He also spent a sabbatical year at the physics department at Cornell University. His research focuses on the magnetic properties at ultralow temperatures of various systems including molecular magnets, heavy fermions, and exotic superconductors. 